\title{
Non-Covalent Functionalization of Carbon Nanotubes by Phthalocyanines Analyzed by Spatial-Resolved EELS
}

\author{
R. Arenal ${ }^{1,2}$, L. Alvarez ${ }^{3}$ J.-L. Bantignies ${ }^{3}$ \\ 1. Laboratorio de Microscopias Avanzadas (LMA), Instituto de Nanociencia de Aragon (INA), \\ Universidad de Zaragoza, Zaragoza, Spain \\ 2. ARAID, Zaragoza, Spain \\ 3. Laboratoire Charles Coulomb, U. Montpelier - CNRS, Montpellier, France
}

Functionalized carbon nanotubes (C-NT) have a huge interest due to their very promising optoelectronic properties [1-2]. However, local (sub-nanometer/atomic level) studies are still challenging. In particular, chemical detailed analyses of these hybrid systems are lacking, even if they are crucial for improving the understanding of such materials. Spatially-resolved electron energy-loss spectroscopy (SR-EELS) is a very powerful technique for providing rich information at the local scale on complex hybrid nanostructures [1-7]. Herein, we will present a detailed and direct investigation at the atomic level, of non-covalent functionalized ( $\pi$-stacked and endohedral) single-walled (SW) C-NTs.

SR-EEL spectra were recorded using a FEI Titan Low-Base microscope, working at $80 \mathrm{kV}$, which is equipped with a Cs probe corrector and ultra-bright XFEG electron source. The HRTEM studies have been carried out in a FEI Titan Cubed microscope (equipped with a Cs image corrector) and also operated at $80 \mathrm{kV}$. Furthermore, in order to avoid electron beam damage, all these measurements have been performed using a liquid-nitrogen-cooled cryo-holder $\left(-170^{\circ} \mathrm{C}\right)$, and relatively low electron doses.

Figure 1 shows some of these results, in particular the case of endohedral functionalization: ironphthalocyanine $(\mathrm{Fe}-\mathrm{Pc})$ moieties in single-walled carbon nanotubes. Figure 1 (a) corresponds to an atomic-sketch showing the supramolecular order of the Fe-Pc within a SWNT and in inset of Figure 1 (e), an atomic-sketch of one of these Fe-Pc molecules is displayed (a Fe atom is surrounded by 4 pyrrolic-like subunits). The HRTEM image of Figure 1 (b) displays one of these individual filled singlewalled C-NTs. These NTs tend to be organized in bundles. Figures 1 (c) and (d), which correspond to of STEM (BF and HAADF) micrographs, show one these bundles of SW-CNTs. A 24x12 EELS spectrumimage (SPIM) has been recorded in the green marked area on one of these NT. (e) EEL spectra extracted from the squared regions marked in Figure 1(d). They correspond to four EEL spectra each of them. C$\mathrm{K}, \mathrm{N}-\mathrm{K}$ and $\mathrm{Fe}-\mathrm{L}_{2,3}$ edges are clearly seen. From these spectra, nitrogen and iron elemental maps can be obtained, see Figure 1 (f). These studies confirm the supramolecular organization of the organic moieties (in this present case showed in this figure, iron-phthalocyanines).

In summary, these works provide very rich information about these hybrid and complex nanomaterials, opening fascinating perspectives for optoelectronic applications of such nanosystems. All these aspects will be discussed in this contribution [8].

References:

[1] A. Setaro et al, Nature Comm. (2017).

[2] L. Alvarez, et al, JPCC 119 (2015), p. 5203.

[3] P. Ayala et al, Rev. Mod. Phys. 82 (2010). 
[4] R. Arenal, O. Stephan, Chap. 5, Eds. L. Francis, A. Mayoral, and R. Arenal, “Advanced TEM: Applications to Nanomaterials", Springer (2015).

[5] R. Arenal, X. Blase, A. Loiseau, Advances in Physics 59 (2010), p. 101.

[6] R. Arenal et al, ACS Nano 7 (2013), p. 4006.

[7] L. Alvarez et al, J. Physical Chem. C, 115 (2011), p. 11898.

[8] The HR-(S)TEM and EELS studies were conducted at the Laboratorio de Microscopias Avanzadas, Instituto de Nanociencia de Aragon, U. Zaragoza, Spain. The Government of Aragon, and the European Social Fund are gratefully acknowledged. R.A. gratefully acknowledges the support from the Spanish Ministry of Economy and Competitiveness (MINECO) through project grant MAT2016-79776-P (AEI/FEDER, UE). The research leading to these results has received funding from the EU H2020 programs ETN Grant Agreement 642742 and "Graphene Flagship" Grant Agreement 696656.
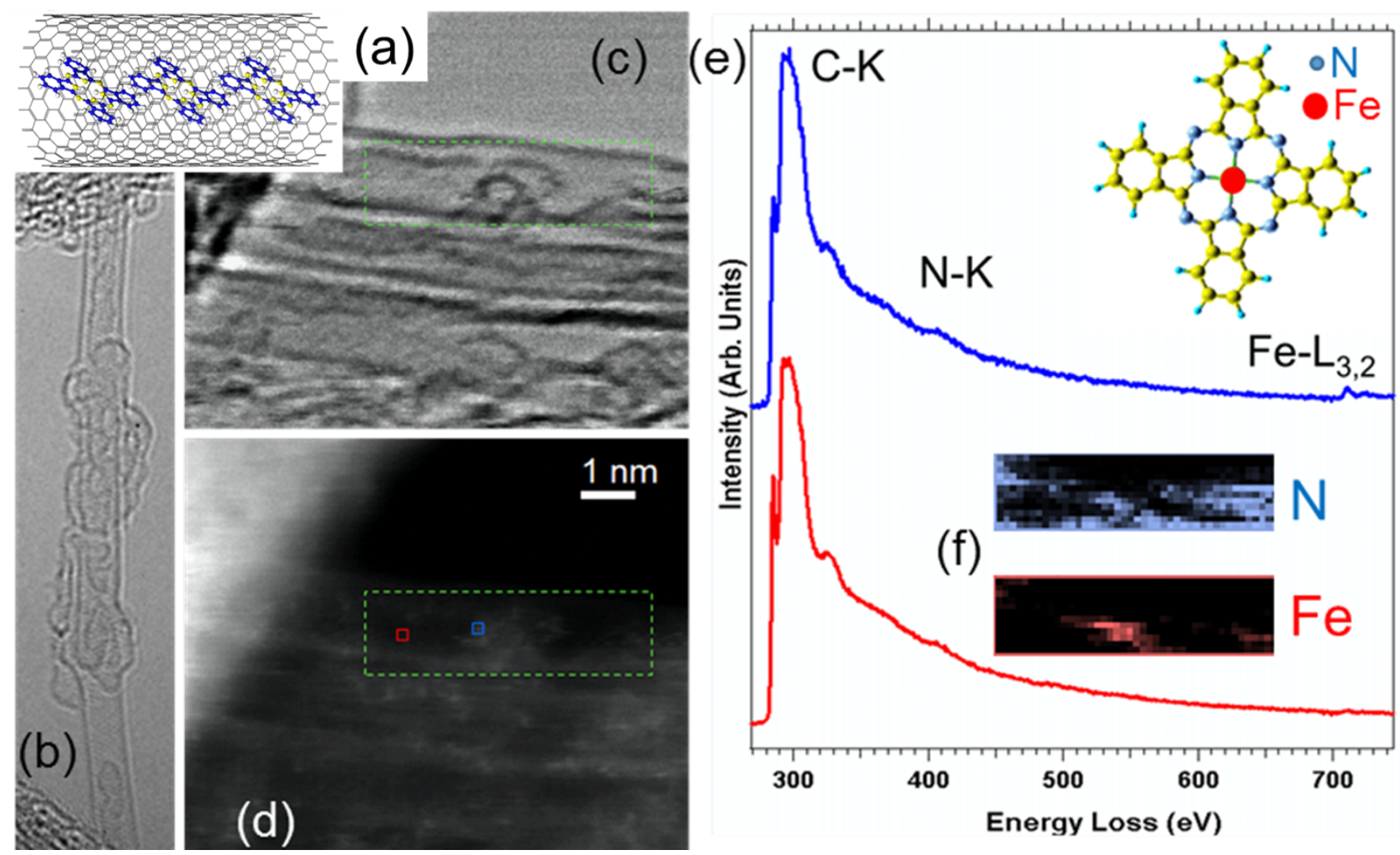

Figure 1. (a) Atomic-sketch showing the supramolecular order of the iron-phthalocyanine (Fe-Pc) moieties within a SW-NT. (b) HRTEM micrograph of a filled SWNT. (c)-(d) BF- and HAADF-STEM images of a bundle of filled SWNT. A SPIM-EELS has been recorded in the green marked area. (e) EEL spectra extracted from the squared regions marked in Fig. 1(d). They correspond to 4 EEL spectra each of them. C-K, N-K and Fe- $\mathrm{L}_{2,3}$ edges are clearly seen. (f) $\mathrm{N}$ and Fe elemental maps. Inset, atomic-sketch of a Fe-Pc (a Fe atom is surrounded by 4 pyrrolic-like subunits). 